\title{
Practical Aspect of Monitoring Hypertension Based on Self-measured Blood Pressure at Home
}

\author{
Yutaka IMAI, Takayoshi OHKUBO*, Masahiro KIKUYA* and Junichiro HASHIMOTO*
}

\begin{abstract}
Devices for home blood pressure (BP) measurement are produced worldwide at a rate of more than $\mathbf{1 0}$ million a year and 30 million such devices have already been distributed in Japan. The clinical significance of home BP measurement is obvious; patients can recognize the effects of antihypertensive treatment. Home BP measurements encourage medication compliance, follow-up clinic visits, and active participation in the medical treatment, thus resulting in improved management of hypertension. Home BP measurements more accurately reflect damage to target organs and the prognosis of cardiovascular diseases. The purpose of home BP measurements is to obtain information on the patient's inherent BP pattern using longterm, repetitive measurement under controlled conditions. Since home BP is measured under controlled condition, values are reproducible, and thus, useful in the diagnosis and treatment of hypertension. Blood pressures measured under standardized condition are indispensable when comparing data among individuals, among groups and among institutes. Working Group of Japanese Society of Hypertension (JSH) established JSH Guidelines for Self-Monitoring of Blood Pressure at Home in 2003. Standardization of the measurement procedure may elevate the position of home BP measurements for the purpose of diagnosing and treating hypertension. As a result, home BP measurements may improve the accuracy of screening for hypertension and assessment of BP control during treatment and encourage drug compliance. Home BP measurements, under such controlled conditions, should have a beneficial effect on the economics of diagnosing and treating hypertension.

(Internal Medicine 43: 771-778, 2004)
\end{abstract}

Key words: blood pressure, home measurements, drug effect, compliance, guidelines

\section{Introduction}

Devices for home blood pressure (BP) measurement are produced worldwide at a rate of more than 10 million a year and 30 million such devices have already been distributed in Japan. The clinical significance of home BP measurement is obvious; patients can monitor the effects of antihypertensive treatment and obtain objective information on medication response. Patients can also recognize elevations of BP when they discontinue or fail to take routine doses of medication. The immediate feedback of home BP measurements encourages medication compliance, follow-up clinic visits, and active participation in medical treatment, thus resulting in improved management of hypertension.

Recent guidelines for the treatment of hypertension such as the Sixth and Seventh Reports of the Joint National Committee on Prevention, Detection, Evaluation and Treatment of High Blood Pressure (JNC-VI and 7) $(1,2)$, the 1999 World Health Organization-International Society of Hypertension (WHO-ISH) Guidelines for the Management of Hypertension (3), the 2003 European Society of Hypertension-European Society of Cardiology (ESH-ESC) Guidelines for the Management of Arterial Hypertension (4), and the Japanese Society of Hypertension (JSH) Guidelines for the Management of Hypertension (5) have all emphasized the importance of home BP measurements in clinical applications of practice, research, and epidemiology. Home BP measurements more accurately and reliably reflect target organ damage and the prognosis of cardiovascular disease. These guidelines include the reference values of hypertension and normotension for home BP measurements. However, none of the guidelines have defined measurement procedure for home BP.

The purpose of home BP measurements is to obtain information on the patient's inherent BP pattern using long-term, repetitive measurement under controlled conditions. Since home BP is measured under controlled conditions, values are

\footnotetext{
From the Department of Clinical Pharmacology and Therapeutics and *the Department of Planning for Drug Development and Clinical Evaluation, Tohoku University Graduate School of Pharmaceutical Science and Medicine, Sendai

Received for publication April 22, 2004; Accepted for publication May 14, 2004

Reprint requests should be addressed to Dr. Yutaka Imai, Department of Clinical Pharmacology and Therapeutics, Tohoku University Graduate School of Pharmaceutical Science and Medicine, Tohoku University Hospital, 1-1 Seiryo-cho, Aoba-ku, Sendai 980-8574
} 
reproducible, and thus, useful in the diagnosis and treatment of hypertension. Blood pressures measured under standardized conditions are indispensable for comparing data among individuals, among groups and among institutes. However, such standards for the measurement of home BP have not been fully established. The Working Group of JSH established the JSH Guidelines for Self-Monitoring of Blood Pressure at Home in 2003 (6). This review referred to the practice aspect of monitoring hypertension based on selfmeasured BP at home and also to the JSH Guideline for home BP measurements.

\section{History of Home Blood Pressure Measurements}

In 1896, Riva-Rocci developed the indirect arm-cuff method for the measurement of BP (7), and in 1905 Korotkoff introduced the use of auscultation in conjunction with the indirect method (8). Since then, the indirect method for BP measurement has remained essentially unchanged for 100 years. Over the past 100 years, BP has been measured in the clinic or other medically oriented settings and has been called casual-clinic BP (CBP). Since the development of indirect BP measurement, hypertension research and treatment methodologies have substantially advanced. The gold standard of BP measurement for practice and research has been CBP. However, an alternative to the CBP was proposed soon after the introduction of indirect BP measurements. The rationale for BP measurement outside the clinical setting is based on the acknowledged and marked variability of BP. Time-dependent and incidental BP variations are well known phenomenon since the 18th century when Stephen Hale observed such variabilities. Clinically, Bevan et al initially demonstrated marked and time-dependent variability of BP in an unrestricted human male subject using direct, continuous BP monitoring for 24 hours (9). In 1940, Ayman and Goldshine reported the concept of "self-BP measurement" and demonstrated an apparent difference between the CBP and the self-measured BP (10). Initially, self-measurement was done using the auscultation method. In the 1970s, an electric device based on the microphone method was marketed, but not widely distributed because of high price, mechanical difficulties, and the issue of auscultation gap. Explosive distribution of home measurement devices since the 1980s is mediated by the development of devices based on the cuff-oscillometric principle.

\section{The Problems of Home Blood Pressure Measurements}

Although the mercury column sphygmomanometer with auscultation is becoming obsolete, we should remember that the gold standard for clinical practice is the Korotkoff sound method using a mercury column sphygmomanometer. The differential properties of the Korotkoff sounds and cuffoscillation lead to an unavoidable difference in BP values between the two methods. The basic algorithm of cuff-oscillo- metric principle has been improved by including procedures to correctly approximate the characteristic changes in cuffoscillation during phase I and phase V Korotkoff sounds. Furthermore, the accuracy of the automatic device is determined by comparison with the auscultation method, and no other standard method is currently available for this purpose. The issue here is the subjectivity and the possible inaccuracy of auscultation when the auscultation method is used as a standard.

Since BP measurements in clinical settings are now primarily obtained by cuff-oscillometric devices, it is inevitable that cuff-oscillometric devices be used in home BP measuring systems. The accumulation of clinical and epidemiological data obtained by authorized cuff-oscillometric devices may finally validate the efficacy of these tools for clinical decision making.

At present, three types of electrical devices for home BP measurements are commercially available: the arm-cuff device, the wrist-cuff device, and the finger-cuff device. Ten million such electrical devices are produced each year in the Far East (including Japan, Korea, Taiwan and China), which represents $85 \%$ of the world production (11). Of those, 35\% are wrist-cuff devices (11). Previously, finger-cuff devices commanded a considerable portion of the market share due to their convenience and ease-of-use. However, it is now apparent that finger BP is physiologically different from brachial BP, and issues of vasospasm in the winter season as well as hydrostatic difference are inevitable. Therefore, manufacturers have now decreased production of finger-cuff devices and extensively increased production of wrist-cuff devices. In Japan, wrist-cuff devices possess $30 \%$ of the market share (12). Wrist-cuff devices are much easier to handle and more portable, but include serious shortcomings. The most important issue is the necessity for correction of the hydrostatic pressure. The reference level for BP measurement is the right atrium. When the measurement site is $10 \mathrm{~cm}$ below (above) the right atrium, systolic BP (SBP) and diastolic BP (DBP) are measured $7 \mathrm{mmHg}$ higher (lower) than those at the level of the right atrium. Even after appropriate correction of the hydrostatic pressure, another issue remains concerning the anatomy of the wrist (11). At the wrist, the radial and ulnar arteries are surrounded by the radial bone, the ulnar bone and several long tendons, including the palmaris longus tendon. Therefore, even a sufficient amount of cuff pressure over the arterial area does not necessarily occlude these arteries completely. As a result, wrist-cuff devices sometime provide erroneous readings, especially for SBP (11). Therefore, arm-cuff devices based on the cuffoscillometric method are recommended for home BP measurement (1-6).

\section{Practical Aspect of Monitoring Hypertension based on Home Blood Pressure Measurements}

Home BP measurements and ambulatory BP monitoring are characterized by increased measurement frequency, and 
Self-measured Blood Pressure

Table 1. Characteristics of Casual, Ambulatory, and Home Blood Pressure Measurements

\begin{tabular}{|c|c|c|c|}
\hline & $\begin{array}{c}\text { Casual BP } \\
\text { (office, clinic, screening) }\end{array}$ & Ambulatory BP & Home BP \\
\hline Characteristic & $\begin{array}{l}\text { including reactive } \\
\text { pressor response }\end{array}$ & $\begin{array}{l}\text { measurements under } \\
\text { several psychological } \\
\text { and physical conditions }\end{array}$ & $\begin{array}{c}\text { measurements under } \\
\text { relatively stable } \\
\text { condition }\end{array}$ \\
\hline Measurement bias & + & $\begin{array}{lll}1 & -\end{array}-$ & $-\sim_{ \pm}$ \\
\hline Measurement frequency & few & many & many \\
\hline Estimation of circadian BP variation & impossible & possible & partially possible \\
\hline Estimation of night-time BP & impossible & possible & possible \\
\hline Estimation of short-term BP variation & impossible & adequate & inadequate \\
\hline Estimation of long-term BP variation & inadequate & inadequate & adequate \\
\hline Reproducibility & poor & poor fair & good \\
\hline Estimation of drug effect & $\begin{array}{l}\text { insufficient due to placebo } \\
\text { effect }\end{array}$ & $\begin{array}{l}\text { occasionally insufficient } \\
\text { due to regression to } \\
\text { the mean }\end{array}$ & adequate \\
\hline Estimation of duration of drug action & impossible & possible & adequate \\
\hline Estimation of drug resistance & inadequate & adequate & adequate \\
\hline $\begin{array}{l}\text { Estimation and diagnosis of white-coat } \\
\text { effect (hypertension) }\end{array}$ & impossible & adequate & adequate \\
\hline $\begin{array}{l}\text { Estimation of paroxysmal hypertension } \\
\text { or episodic hypotension }\end{array}$ & impossible & adequate & occasionally possible \\
\hline
\end{tabular}

thus, increased information on BP. In addition, these methods provide BP information in relation to time. Such characteristics of home BP measurements provide advantages and superiority when compared to CBP (Table 1).

Home blood pressure measurement and diagnosis of hypertension

References value of home blood pressure

Although home BP measurement devices are distributed widely throughout the world, the practice of monitoring hypertension using these devices has not been established because of the deficiency of reference values for hypertension and normotension using this home equipment. Another issue is that standardization of home BP measurements has not been established.

Since 1986, we have been conducting an epidemiological survey of hypertension using home BP in Ohasama, in the northern part of Japan. Ohasama initially had a population of 9,400 , but this has now dropped to 6,800 . Over the past 18 years, we have obtained home BP data from 5,000 subjects aged over 7 years, as well as long-term clinical outcomes and information on risk factors and predictors. One of the initial purposes of the study was to define reference values for home BP measurements with respect to prognosis in a longterm prospective study.

Several methods are available for obtaining these reference values. The first involves the distribution criteria, for example mean $+\mathrm{SD}$, mean $+2 \mathrm{SD}$ or 95 th percentile value of the reference population. These values provided us with the distribution of home BP level in the population, but clinical significance of these values is still uncertain.

Another method uses correspondence criteria, which derives home BP levels corresponding to CBP of 140/90 $\mathrm{mmHg}$. However, the relationship between CBP and home $\mathrm{BP}$ has not been defined well enough to obtain accurate corresponding values; the correlation coefficient of the relationship between CBP and home BP has been calculated to be approximately 0.5 (13). However, the linear regression analysis deduced that $140 / 90 \mathrm{mmHg}$ for CBP corresponds to $125 / 80 \mathrm{mmHg}$ for home $\mathrm{BP}$, suggesting that the normative value of home $\mathrm{BP}$ is less than $125 / 80 \mathrm{mmHg}$.

The most meaningful reference values would be provided by a long-term prospective study based on the resultant cardiovascular morbidity and mortality. Several observational and interventional studies are currently ongoing worldwide. The Ohasama study was initiated first and is the only study aiming to provide such reference values. Subjects from the Ohasama population aged 40 years and over were followed up for an average of 10.6 years. Home BP and CBP values were classified equally into quintiles on the basis of BP level. The relationship between BP level and stroke incidence being analyzed by a Cox regression model was adjusted for age, sex, and drug treatment. No specific tendency was observed in CBP. In subjects in the highest quintile of home BP $(\geqq 135 / 85 \mathrm{mmHg})$, a significant increase in relative hazard was observed, suggesting the higher predictability of home BP when compared with CBP (14) (Fig. 1).

These results obtained from Ohasama studies were cited in the JNC-VI (1) and 1999 WHO-ISH guidelines (3) and were the basis of reference values (Table 2) for home BP measurements given in these guidelines.

Definition of white-coat hypertension and white-coat effect White-coat hypertension - reproducible hypertension in 


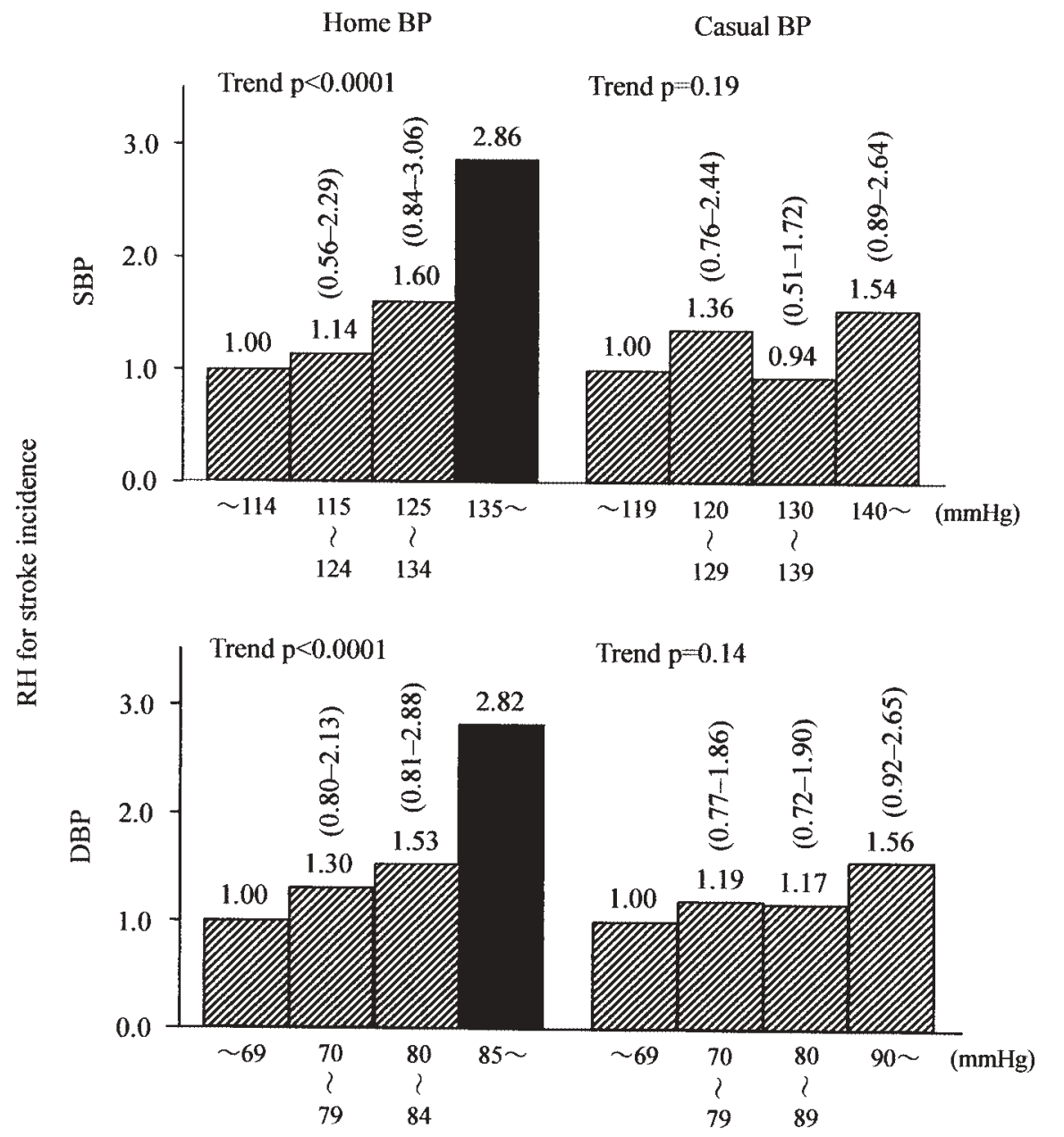

Figure 1. Association between home and casual-screening blood pressure (BP) values and stroke risk. Relative hazards (RH) and $95 \%$ confidence intervals (CI) of home and casual-screening systolic BP (SBP) and diastolic BP (DBP) level adjusted for age, gender, smoking status, the use of antihypertensive medication, history of heart disease, hypercholesterolemia, and diabetes for first symptomatic stroke. The group with the lowest risk was treated as the reference category $(\mathrm{RH}=1)(14)$.

Table 2. Reference Values of Home BP Value by JNC VI and WHO/ISH

\begin{tabular}{lcc}
\hline & $\begin{array}{c}\text { HBP value } \\
(\text { Ohasama }) \\
(\mathrm{mmHg})\end{array}$ & $\begin{array}{c}\text { Reference } \\
\text { value } \\
(\mathrm{mmHg})\end{array}$ \\
\hline $\begin{array}{l}\text { Hypertension } \\
\text { Cox model (non-parametric) }\end{array}$ & $\geq 138 / \geq 83$ & $135 / 85$ \\
Normotension & & \\
$\begin{array}{l}\text { Cox model (non-parametric) } \\
\text { Corresponding value of 140/90 } \\
\text { (Clinic BP) }\end{array}$ & $120-127 / 72-76$ & $123 / 77$ \\
$\begin{array}{l}\text { Mean home BP value + 1SD } \\
\text { (with normal Clinic BP value) }\end{array}$ & $125 / 77$ & $\begin{array}{c}\text { (WHO/ISH) } \\
\text { (WN }\end{array}$ \\
\hline
\end{tabular}

medical settings and normotension in non-medical settings is accurately defined using the normative value of home BP, i.e., CBP equal to or higher than $140 / 90 \mathrm{mmHg}$ and home BP less than $125 / 80 \mathrm{mmHg}$. The Ohasama study examined the prognostic significance of white-coat hypertension (15). According to the Cox regression model, the relative hazard in white-coat hypertensive patients was similar to that seen in true normotensive subjects, whereas true hypertension and reversed white-coat hypertensive subjects (hypertension in the non-medical setting and normotension in the medical setting) carried a significantly higher relative hazard for cardiovascular mortality (Fig. 2) (15). However, recent analysis demonstrated that the development of sustained hypertension was more frequent in patients with white-coat hypertension than in those with true normotension during a 10 -year observation period, suggesting that white-coat hypertension is a 


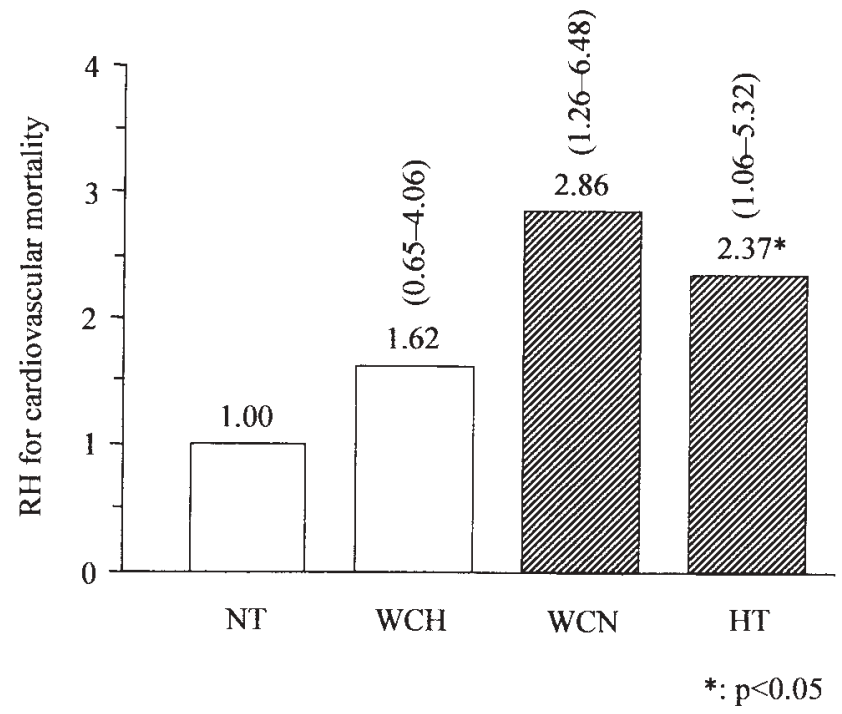

Figure 2. Risk of white-coat hypertension, white-coat normotension and sustained hypertension for cardiovascular mortality. Relative hazard (RH) for cardiovascular mortality and $95 \%$ confidential intervals (CI). NT: normotension, WCT: whitecoat hypertension, WCN: white-coat normotension (masked hypertension), HT: sustained hypertension. Normotension was treated as the reference category $(\mathrm{RH}=1)(15)$.

S.S (M) 59 y.o.

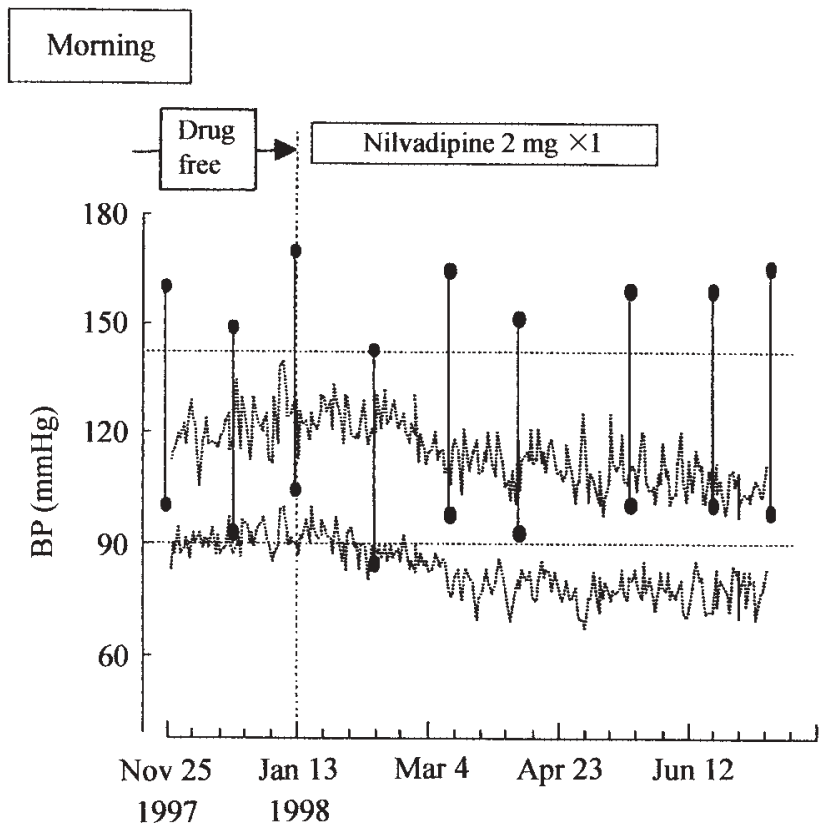

(Date) benign condition during short-term observation periods but it becomes a cardiovascular risk during long-term observation periods.

Definition of the white-coat effect leads to the diagnosis of resistant hypertension or intractable hypertension. For example, in cases of essential hypertension, patients whose CBP was continuously higher than 160/100 $\mathrm{mmHg}$ and home BP was higher than $135 / 90 \mathrm{mmHg}$ were treated with a calcium antagonist. The antihypertensive effect of the drug was never observed in CBP, while the drug sufficiently decreased home BP (Fig. 3). The clinical significance of home $\mathrm{BP}$ is apparent from this case; the white-coat effect is resistant to an antihypertensive regimen.

Circadian blood pressure variation and home blood pressure measurements

Recently, circadian BP variation has received attention as a risk factor in cardiovascular diseases. In the Ohasama study, non-dipper and inverted dipper circadian BP variations were apparent cardiovascular risk factors. However, the so-called extreme-dipper circadian BP variation was a benign condition (16). Such BP information was obtained only by ambulatory BP monitoring. Recently, we developed new equipment for self-BP measurements which allows monitoring of BP during sleep (17). Thus, the nocturnal BP level is
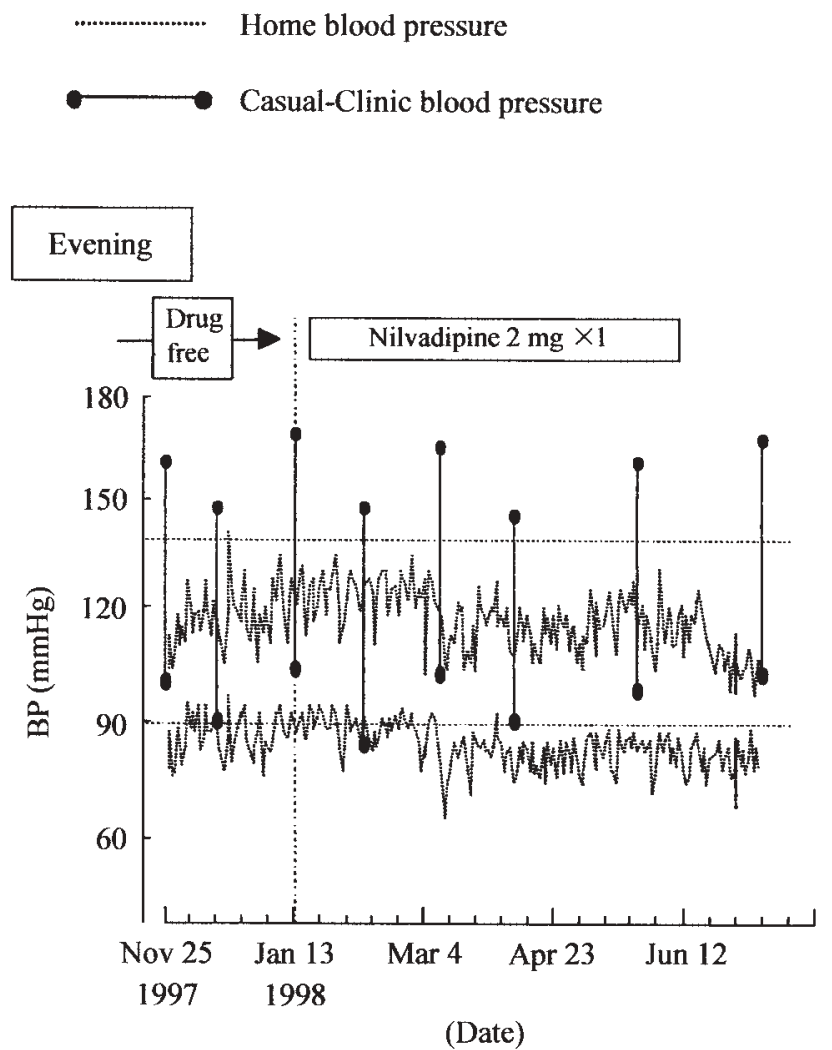

Figure 3. Effects of nilvadipine, a calcium antagonist, on white-coat effect in a hypertensive patient. Nilvadipine did not decrease casual-clinic blood pressure (CBP), but decreased home BP both in the morning and evening. SBP: systolic BP, DBP: diastolic BP, HBP: home BP, HR: heart rate. 
now available from home BP measurements. However, the most common routine for home BP measurements is in the morning and in the evening.

Recently, the concept of hypertension in the morning is an issue in the management of hypertension. Morning hypertension is mediated by a morning surge and non-dipper or inverted dipper circadian BP variation. The morning surge of BP represents a mirror image of nocturnal dipping, and thus, is essentially observed in extreme dippers. In the Ohasama study, morning hypertension is primarily mediated by nondipper or inverted dipper circadian BP variation and only $10 \%$ of patients with morning hypertension have an extreme dipper pattern, and thus, a morning surge. Recently, Kamoi et al reported that in normotensive (per CBP) patients with diabetes mellitus only those with high $\mathrm{BP}$ in the morning obtained during home BP measurements had severe target organ damage (18). It is well known that patients with diabetic target organ damage usually have non-dipper or inverted dipper circadian BP variation, suggesting that the morning hypertension reported by Kamoi et al reflects the overall BP load throughout 24 hours. Morning hypertension reported by Kamoi et al is also defined as reverse white-coat hypertension or white-coat normotension, which was related to poor prognosis in the Ohasama study (15). This concept was later reported as masked hypertension by Pickering et al (19). We found that masked hypertension is mediated by non-dipper circadian BP variation, inverted dipper circadian $\mathrm{BP}$ variation and insufficient duration of action of the antihypertensive medication (20). Home BP measurement is the only a practical method to determine the occurrence of morning hypertension.

\section{Day-by-day variability of blood pressure}

Short-term BP variability is a risk factor for cardiovascular diseases (21). Such short-term information is available from ambulatory BP monitoring, while the information on day-by-day variability is obtained only with home BP measurements. The Ohasama study demonstrated that day-by-day variability reflects the risk of cardiovascular diseases. Thus, home BP measurements can now replace ambulatory BP monitoring.

\section{Treatment of hypertension based on home blood pres- sure measurements}

Evaluation of antihypertensive effect

Since home BP is measured under controlled conditions using a standardized method, the reproducibility of home BP is assured and no placebo effect is observed in the measurements (22). Therefore, the accuracy and validity of home BP measurements reflects the physiological response to the clinical pharmacology of antihypertensive drugs; e.g., a decrease in systolic BP by $6 \mathrm{mmHg}$ is determined by 15 subjects when based on the home BP measurements. Home BP measurement improves the quality in clinical pharmacological studies.

\section{Evaluation of duration of action of antihypertensive effect}

Home BP measurements can be used to evaluate medication effects and the duration of action. Figure 4 demonstrates that when trichlomethiazide was administered, once in the morning, home BP was decreased when measured before taking the next dose; this suggested that the duration of action for this drug is more than 24 hours. Such characteristics of home BP measurements provide an index of duration of action of drugs, i.e., the morning effect vs. evening effect ratio $(\mathrm{M} / \mathrm{E}$ ratio), which is comparable to trough/peak $(\mathrm{T} / \mathrm{P})$ ratio obtained by ambulatory $\mathrm{BP}$ monitoring. The M/E ratio is more reliable than the T/P ratio, since the former is obtained by the average of multiple measurements of the difference between the period before treatment and the treated period.

\section{JSH Guidelines for self-monitoring of blood pressure at home}

Home BP measurements are indispensable for the improvement of management of hypertension in medical practice as well as for the recognition of hypertension in the population. Therefore, practice of self-measurement of BP is the first priority and for this purpose it is not necessarily expected that strict measurement conditions will be set. However, the presence of a standard for home BP measurements may be convenient and useful for practitioners as well as for patients. Such standards should be intended to instruct patients and subjects in the general population on how to measure BP at home and may provide a shared basis of information for clinical decision making. The Working Group for Establishment of Guidelines for Measurement Procedure of Self-Monitoring of Blood Pressure at Home of the JSH has established standard for all techniques and procedures of home BP measurements (6).

The recommendations are as follows:

1) For home BP use, arm-cuff devices are recommended. They should be based on the cuff-oscillometric method, validated officially, and confirmed for accuracy in each individual.

2) BP should be measured in the upper arm. Finger-cuff devices and wrist-cuff devices should not be used for home BP measurements.

3) Devices for home BP measurement should be adapted from the American Association Medical Instrumentation (AAMI) standard and the British Hypertension Society (BHS) guidelines. In addition, the difference between the BP measured by the auscultatory method and that measured using the device should be $5 \mathrm{mmHg}$ or less in each individual. Accuracy and function of the home measurement device should be validated before use and at regular intervals.

4) Home BP should be monitored under the following conditions.

The morning measurements:

- within 1 hour after waking 

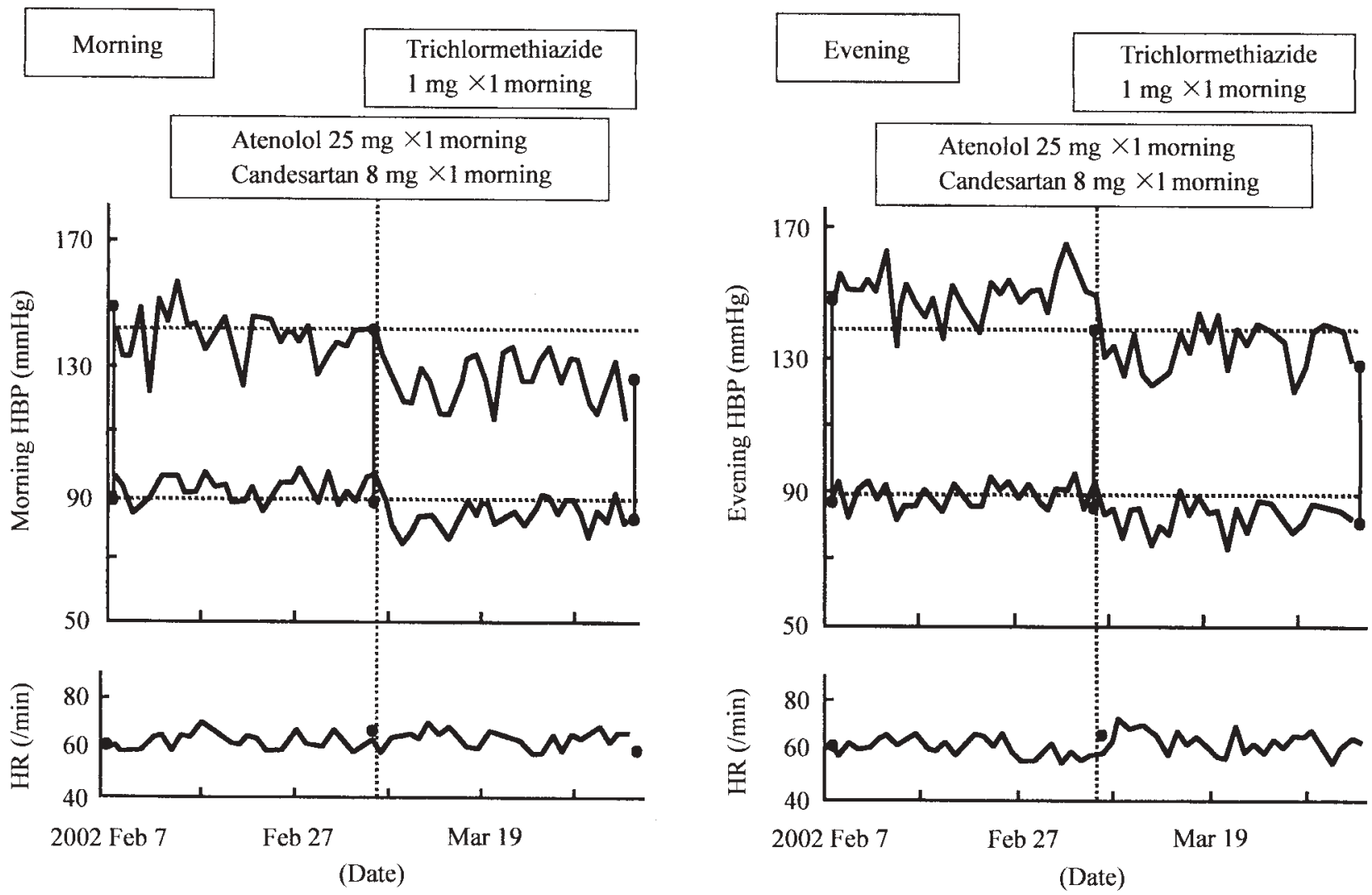

Figure 4. Effect of trichlormethiazide, administered once in the morning, on home blood pressure (HBP) in the morning and evening in a patient with essential hypertension. The HBP in the morning was measured before morning dose, thus, reflecting that the duration of action of thiazide is over 24 hours.

- after micturition

- after 1 to 2 minutes of sitting at rest

- before drug ingestion

- before breakfast

The evening measurements:

- just before going to bed

- after 1 to 2 minutes of sitting at rest

5) Home BP should be measured at least once in the morning and once in the evening.

6) All home BP measurements should be documented without selection or omission and include the date, time, and pulse rate. Use of a device with a printer or an integrated circuit memory is useful to avoid selection bias.

7) The home BP in the morning and that in the evening should be averaged separately for a certain period. The first measurement on each occasion should be used for totaling.

8) Home BP values that average $135 / 80 \mathrm{mmHg}$ and over, for a certain period, indicate hypertension. Average values of $135 / 85 \mathrm{mmHg}$ and over indicate definite hypertension. Normotension is defined as less than $125 / 80 \mathrm{mmHg}$ and definite normotension as less than $125 / 75 \mathrm{mmHg}$.

The guidelines aimed to establish practical advice which would not restrict casual daily life of the subjects. For example, one to two minutes of rest before measurements would be acceptable by the majority of people who measure BP at home every day. In the guidelines, prohibition of smoking and taking coffee before measurement was not addressed. Since BP values obtained after smoking and taking coffee would reflect daily behavior and lifestyle, regulation may actually interfere with the validity of the BP readings. Room temperature was also not addressed by the guidelines, since casual temperature per se is an important factor for daily BP level. In the present guidelines, it has been emphasized that home BP should be routinely measured at least once per occasion. "At least once" means that more than one measure- 
ment during that occasion is also permissible. Actually subjects measure their BP repeatedly, until a reasonable value is obtained when their home BP is high. We must evaluate all values recorded. However, to compare data among individuals, groups and institutes a standardized measurement is necessary. The first measurement would be considered a common denominator in all cases. Therefore, the average of the first measurement for a certain period is an important commonality for clinical decision making.

\section{Conclusion}

At present, international reference values have been established. However, the treatment goal for home BP level has not yet been established. The normotensive value of home $\mathrm{BP}$ is set at the level of $125 / 80 \mathrm{mmHg}$. This value is approximately equivalent to a CBP level of $140 / 90 \mathrm{mmHg}$. Therefore, it seems that a value of less than $125 / 80 \mathrm{mmHg}$ would be the goal for home BP. However, setting the goal for home BP must be based on the results of large-scale intervention studies. Among such studies the Hypertension Objective Treatment on Measurements by Electrical Device of BP (HOMED-BP) study is ongoing in Japan (23)

Standardization of the measurement procedure may elevate the position of home $\mathrm{BP}$ measurements in the practice of diagnosing and treating hypertension, and as a result, home BP measurements may bring greater accuracy in the screening for hypertension and assessment of BP control during treatment and improved drug compliance. Home BP measurements under such controlled conditions are expected to have a beneficial effect on the economics of the diagnosis and treatment of hypertension.

\section{References}

1) National High Blood Pressure Education Program. The Sixth Report of the Joint National Committee on Prevention, Detection, Evaluation, and Treatment of High Blood Pressure. Arch Intern Med 157: 24132446, 1997.

2) Chobanian AV, Bakris GL, Black HR, et al. The National High Blood Pressure Education Program Coordinating Committee. The Seventh Report of the Joint National Committee on Prevention, Detection, Evaluation, and Treatment of High Blood Pressure. The JNC 7 Report. JAMA 289: 2560-2572, 2003.

3) Guidelines Subcommittee. 1999 World Health Organization-International Society of Hypertension Guidelines for the Management of Hypertension. J Hypertens 17: 151-183, 1999.

4) Guideline Committee. 2003 European Society of Hypertension-European Society of Cardiology guidelines for the management of arterial hypertension. J Hypertens 21: 1011-1053, 2003.

5) Guidelines Subcommittee for the Japanese Society of Hypertension. Japanese Society of Hypertension Guidelines for the Management of Hypertension (JSH 2000). Japanese Society of Hypertension 2000.

6) Imai Y, Otsuka K, Kawano Y, et al. Japanese Society of Hypertension (JSH) Guidelines for Self-Monitoring of Blood Pressure at Home. Hypertens Res 26: 771-782, 2003.

7) Riva-Rocci S. Un nuovo sfigmomanometro. Gazz Med Torino 47: 981-1001, 1896.

8) Korotkoff N. To the question of methods of determining the blood pressure. Rep Imp Mil Acad 11: 365-367, 1905.

9) Bevan AT, Honour AJ, Stott FH. Direct arterial pressure recording in unrestricted man. Clin Sci 36: 329-344, 1969.

10) Ayman D, Goldshine A. Blood pressure determinations by patients with essential hypertension. Am J Med Sci 200: 465-474, 1940.

11) Kikuya M, Chonan K, Imai $Y$, et al. Accuracy and reliability of wristcuff devices for self measurement of blood pressure. J Hypertens 20: 629-638, 2002.

12) Shirasaki O, Terada H, Niwano $K$, et al. The Japan Home-Health Apparatus Industrial Association: investigation of home-use electronic sphygmomanometers. Blood Press Monit 6: 303-307, 2001.

13) Imai $Y$, Satoh H, Nagai K, et al. Characteristics of community based distribution of home blood pressure in Ohasama, in northern Japan. J Hypertens 11: 1441-1449, 1993.

14) Ohkubo T, Asayama K, Kikuya M, et al. How many times should blood pressure be measured at home for better prediction of stroke risk? 10-year follow-up results from the Ohasama study. J Hypertens 22: 1099-1104, 2004.

15) Ohkubo T, Imai Y, Tsuji I, et al. Prognostic significance for mortality among "white coat" and "reversed white coat" hypertension. Ann Noninvasive Ambulatory Monit 1 (pt2): 212, 1996.

16) Ohkubo T, Imai Y, Tsuji I, et al. Relation between nocturnal decline in blood pressure and mortality: The Ohasama Study. Am J Hypertens 10: 1201-1207, 1997.

17) Chonan K, Kikuya M, Araki T, et al. Device for the self-measurement of blood pressure that can monitor blood pressure during sleep. Blood Press Monit 6: 203-205, 2001.

18) Kamoi K, Miyakoshi M, Soda S, et al. Usefulness of home blood pressure measurements in the morning in type 2 diabetic patients. Diabetes Care 25: 2218-2223, 2003.

19) Pickering TG, Davidson K, Gerin W, Shwartz JE. Masked hypertension. Hypertension 40: 795-796, 2002.

20) Chonan K, Hashimoto J, Ohkubo T, et al. Insufficient duration of action of antihypertensive drugs mediates high blood pressure in the morning in hypertensive population: The Ohasama Study. Clin Exp Hypertens 24: 261-275, 2002.

21) Kikuya M, Hozawa A, Ohokubo T, et al. Prognostic significance of blood pressure and heart rate variabilities. Hypertension 36: 901-906, 2000.

22) Imai Y, Ohkubo T, Hozawa A, et al. Usefulness of home blood pressure measurements in assessing the effect of treatment in a single-blind placebo-controlled open trial. J Hypertens 19: 179-785, 2001.

23) Fujiwara $T$, Nishimura $T$, Ohkuko $T$, et al. Rationale and design of HOMED-BP Study: hypertension objective treatment based on measurement by electrical devices of blood pressure study. Blood Press Monit 7: 77-82, 2002. 